\title{
Temporal manipulation of stimulus patterns using the Apple II: Tachistoscopic and part presentation
}

\author{
I. H. FRASER, J. R. LISHMAN, and D. M. PARKER \\ Kings College, University of Aberdeen, Old Aberdeen, Scotland
}

\begin{abstract}
A technique is described in which line-drawn pictures can be rapidly interlaced, allowing for sequential part presentation or tachistoscopic presentation on the Apple II. This is accomplished using a two-stage process in which the pictorial stimuli are converted into machine code data files and then used to create a rapidly changing display consisting of two or more line drawings. The latter stage could be accomplished in less than $2 \mathrm{msec}$ but is limited by the frame time of the monitor (17-20 msec).
\end{abstract}

In much of the visual research on form perception, the chief mode of presentation involves simultaneous display of all parts of the stimulus form. Although this type of presentation has been adopted as the standard, there are viable alternatives. McFarland (1965), for example, presented arguments supporting a mode of presentation in which the parts of a stimulus are presented in a temporal sequence. He believed that the existing physiological data suggested that the information simultaneously impinging on the retina is processed sequentially or at different rates within the system and, therefore, that perceived simultaneity must be a by-product of the process of figural synthesis. He suggested that sequential part presentation might be helpful in assessing the parameters of the visual pattern recognition mechanism and its tolerance to manipulations.

The existing literature, however, indicates that little research has been conducted utilizing temporal fragmentation as a mode of presentation. One possible explanation for this is that existing equipment is not designed for such presentations, and, although adaptations can be made, there is still a limit as to the number of parts in which a stimulus can be successfully presented. Two examples of adaptations to existing equipment can be found in the studies conducted by McFarland (1965), who, in an attempt to present three sides of a triangle sequentially, devised an apparatus that consisted of three slide projectors fitted with Sylvania R1131c bulbs to allow for rapid presentation. The projectors were controlled by five solidstate timers, and a logic circuit controlled the sequence of part presentation. Although this novel combination of existing pieces of apparatus proved successful in McFarland's study, it is obvious that two extra timers and another projector are required for each additional stimulus part, and spatial alignment of the stimulus parts becomes increasingly problematic as the number of parts

I. H. Fraser and D. M. Parker are with the Department of Psychology and J. R. Lishman is with the Department of Computer Science at Kings College, University of Aberdeen, Old Aberdeen AB9 2UB, Scotland. increases. Eriksen and Collins (1967) utilized a two-field tachistoscope to present two parts of a stimulus over varying interstimulus intervals. At first glance, the tachistoscope seems ideal for stimulus part presentation, but since T-scopes with more than four fields are costly and rare, the variety of studies afforded by this method is rather restricted.

Apparatuses designed specifically for the purpose of sequential stimulus part presentation have been cumbersome (see Ikeda \& Uchikawa, 1978). In an attempt to find another way of successfully sequentially presenting the parts of a stimulus without the restrictions that have plagued past attempts, we turned to the microcomputer (i.e., the Apple IIe).

\section{METHOD}

The BASIC functions available to turn the Apple into a tachistoscope are elementary and restrictive. The most common methods are: (1) Using the two high-resolution screens of the Apple with a low-resolution screen intervening as a blank between exposures. This is simple, but the experimenter is limited to two pictures. (2) Reading in new pictures from disk. This is not difficult, but takes $15 \mathrm{sec}$ per picture. (3) Moving a picture image from elsewhere in memory. This is a slow process, taking about $0.5 \mathrm{sec}$ per image, the rate at which an image "crumbles" after "HGR" (the command to implement highresolution graphics) shows the effect. (4) Changing only what needs to be changed for pictures with a small area to be changed. The changes should be stored as a program in memory; for example,

$$
\begin{aligned}
& \text { LDA }\left\{23 \text {; new picture value }{ }^{1}\right. \\
& \text { STA } 4567 \text {; address in picture space } \\
& \text { RTS ; return to BASIC }
\end{aligned}
$$

This representation is compact for small changes in a picture, does not need to be changed if it is moved in memory, and changes the picture nearly as fast as is possible. (It would go slightly faster if the program loaded 
the value once, then stored that value wherever it was needed.)

Listing 1 is a program that was constructed in an attempt to increase the speed of graphics drawing and is an extended version of item 4 above. One reason for the computer's inability to draw a complex stimulus quickly is that the system has to convert every line of the program from BASIC to machine code. Interpreting one line of BASIC in about $10 \mathrm{msec}$ may seem rather fast. However, when the stimulus is very complex, the time consumed in this line-by-line interpretation adds up. For example, a stimulus made up of 100 lines of BASIC would take about $1 \mathrm{sec}$ to process. If, however, a program is converted into machine code (i.e., compiled) before it is run, this on-going line-by-line interpretation can be avoided, resulting in a large reduction in processing time. The first program performs this task and stores these compiled files on disk. Lines 140-210 show simple outline triangles; a program for a more complex display should be substituted as required by the user. When the program is run, it will first draw the stimulus on both highresolution graphics, screens 1 (line 170) and 2 (line 210), then compare both screens point by point, recording the differences and converting the differences into machine code (lines 200-830). The computer will then allow the user to compare both screens (lines 890-990) and, when the user is satisfied, save the data on a disk under the user's chosen file name (lines 1000 on).

Listing 2 is a program that takes these data files from the disk and stores them in a set of higher memory addresses. One of the problems with presenting information from a disk is that the process can be very timeconsuming, taking as much as $3 \mathrm{sec}$ to retrieve a file, depending on its complexity. However, it is possible to store the files in the computer's memory to obtain shorter access times, but this requires that particular areas of memory be set aside solely for file storage. The two areas capable of this type of manipulation are found in the lower and higher memory addresses (2048-8192 and 24756-38299, respectively, for a $48 \mathrm{~K}$ machine).

The lower available memory addresses are located between the BASIC system (0000-2048) and high-resolution graphics, page 1 (8192-16384). LOMEM (which specifies the lower limit of these memory addresses) is automatically set just above the last line of the currently stored program (which begins loading at location 2049). This means that the amount of memory space available to the graphics files depends on the complexity of the program used to present the files tachistoscopically. Furthermore, variables utilized within a program are also stored in these lower memory addresses, building up from just above the area occupied by the last graphics file. This, too, can create problems by taxing the limits of the lower available memory space, resulting in an overflow into the memory addresses reserved for high-resolution graphics, screen 1. This type of overflow usually results in a residue of short horizontal lines appearing on the video screen.
The higher available memory addresses are located between high-resolution graphics, page 2 (16384-24576), and the DOS operating system (38299-49151). Although these high memory addresses are utilized for the storage of string arrays, they are not affected by the complexity of the currently stored program. Furthermore, due to the fact that high-resolution graphics screen 2 (HGR2) is not used in this particular tachistoscopic program, the string arrays (which build down from the address immediately after the last stored graphics file) can invade the memory allocated to HGR2 without disrupting its performance. Therefore, it seems that the most appropriate place to store complex graphics files is in the higher available memory addresses.

Having avoided the two basic problems alluded to above, it is now possible to present a stimulus within a few milliseconds. This means that the only restriction affecting the stimulus presentation time is the scanning speed of the video monitor, which is approximately 16.6-20.0 msec, depending on whether the main frequency is 60 or $50 \mathrm{~Hz}$.

Listing 2 shows how the displays stored by Listing 1 may be rapidly sequenced. Lines $130-150$ load the compiled file and lines 200-240 find the RTS. Lines 300-320 plot constant parts of the picture, and lines $400-440$ exercise the program.

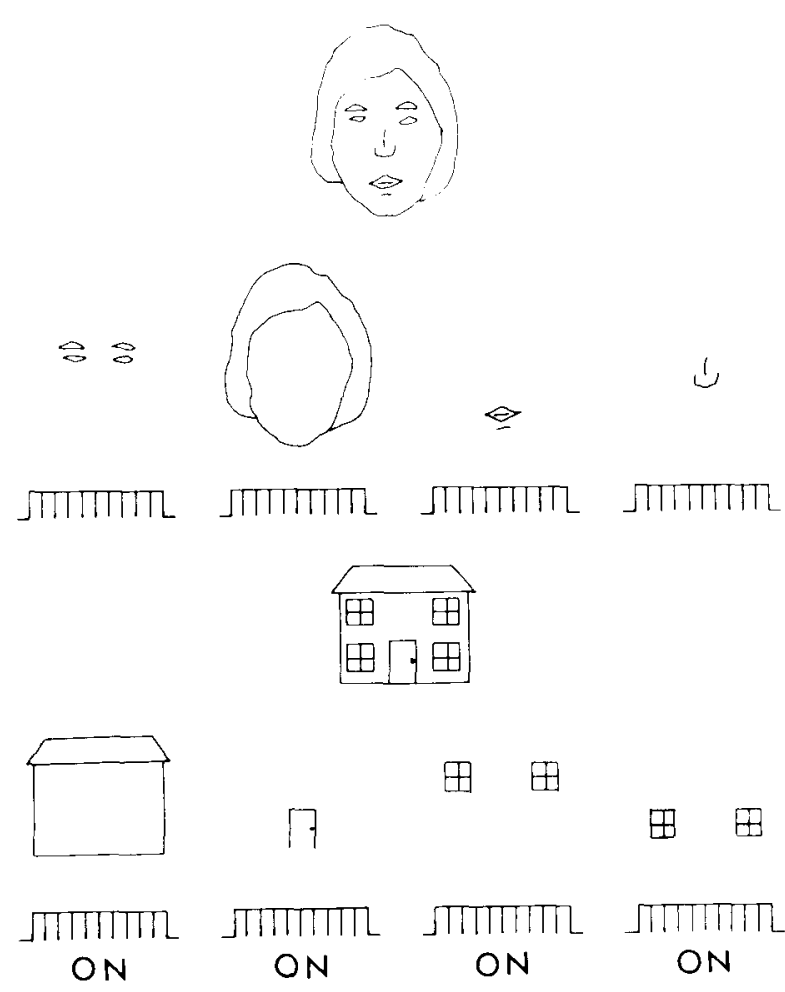

Figure 1. Two typical stimulus patterns used in experiments by the authors: a schematic face and house. The figure shows the complete stimulus and indicates a possible presentation sequence for its four component parts. In the experiments, on-time = off-time = 20 msec. 


\section{CONCLUSION}

In our experiments we have shown to subjects temporally fragmented outline drawings, such as those shown in Figure 1. The results indicate that there are highly significant effects of the order of stimulus parts, even when the rated presentation duration is kept to a maximum of $140 \mathrm{msec}$ (Parker \& Fraser, 1987). Beyond this, the program also has proved to be very flexible in its ability to adapt to many situations, including the analysis of feature salience in pattern recognition (Fraser \& Parker, 1986). An example of this flexibility is the fact that the program can easily be altered so as to present within the same study two or more different complex stimuli, such as those shown in Figure 1. The only restriction is that the combined data files cannot exceed the capacity of the higher free memory addresses. This problem can be avoided by changing the lower limit of the higher available memory addresses in line 300 to include highresolution graphics, page 2 . However, this will only work if the tachistoscopic program does not require much storage of string arrays.

\section{AVAILABILITY}

The program is available to anyone who sends a $51 / 4$ in. disk and reusable mailer to the authors.

\section{REFERENCES}

Eriksen, C. W., \& Collins, J, F. (1967). Some temporal characteristics of visual pattern perception. Joumal of Experimental Psychology, 74, 476-484.

Fraser, I. H., \& Parker, D. M. (1986). Reaction time measures of feature saliency in a perceptual integration task. In H D. Ellis, M. A. Jeeves, F. Newcombe, \& A. Young (Eds.), Aspects of face processing (pp. 45-52). Dordrecht: Martinus Nijhoff.

IKEDA, M., \& UCHIKAWA, K. (1978). Integrating time for visual pattern perception and comparison with the tactile mode. Vision Research, 18, 1565-1571.

MCFARLAND, J. M. (1965). Sequential part presentation: A method of studying visual form perception. British Journal of Psychology, 56, 439-446.

PARKer, D. M., \& Fraser, I. H. (1987). Processing biases in the integration of temporally fragmented patterns. Manuscript in preparation.

\section{NOTE}

1. On versions of the Apple in the United States, the $\mathfrak{E}$ symbol is replaced by the \# symbol.

\section{LISTING 1}

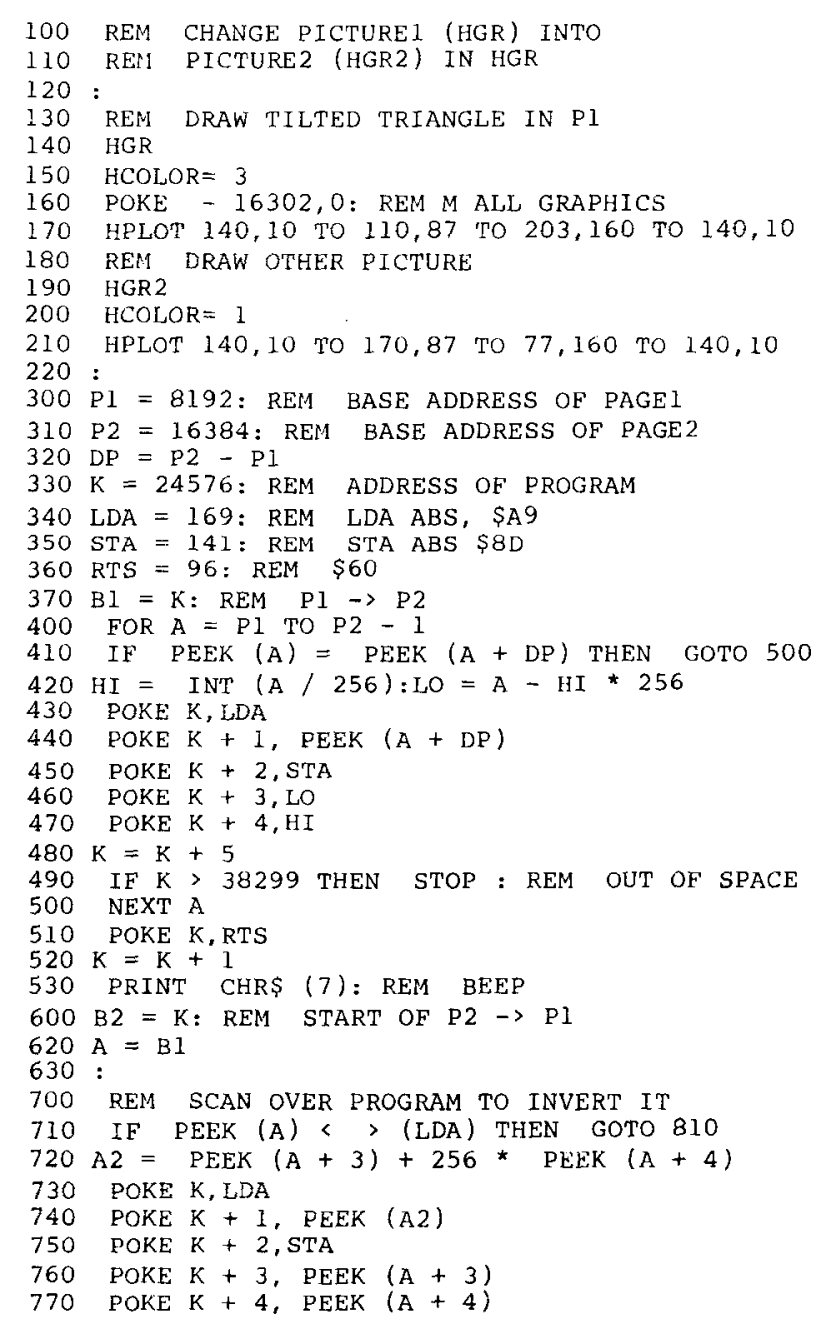




\section{LISTING 1 (Continued)}

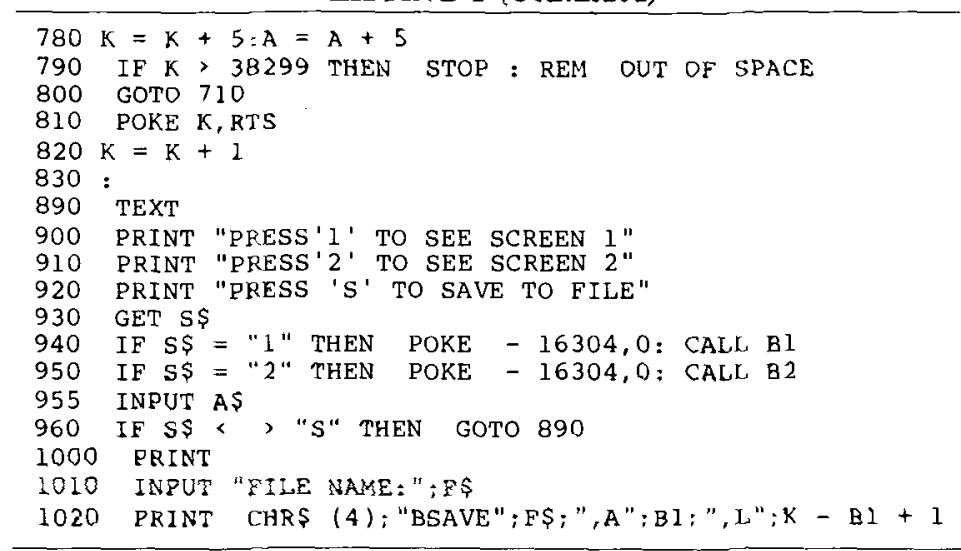

\section{LISTING 2}

100 REM EXAMPLE OF USE

$110 \mathrm{Bl}=16384:$ REM START OF PROGRAM IN HGR2 SPACE

120 RTS $=96:$ REM END OF PROGRAM MARKER

130 REM LOAD THE FILE

140 INPUT "EILE NAME:";F\$

150 PRINT CHRS (4); "BLOAD ";F\$;",A";BI

200 REM FIND ITS END

210 FOR $A=B 1$ TO 32999 STEP 5

220 IF PEEK $(A)=$ RTS THEN B2 = A + I: GOTO 300

230 NEXT $A$

240 STOP : REN END NOT FOUND

300 REM LOAD IN OTHER FILES AFTER THIS ONE

310 HGR : POKE - 16302,0: HCOLOR $=3$

318 REM PLOT CONSTANT PARTS

320 HPLOT 140,10 TO 110,87

400 CALL BI

410 FOR $J:=1$ TO 1000: NEXT J: REM WAIT

420 CALL B2

430 FOR $\mathrm{J}=1$ TO 1000: NEXT J: REM WAIT 440 GOTO 400

(Manuscript received September 19, 1986; revision accepted for publication February 9, 1987.) 